\title{
Role of the Soluble Receptor for Advanced Glycation End Products (sRAGE) as a Prognostic Factor for Mortality in Hemodialysis and Peritoneal Dialysis Patients
}

\author{
Elena Dozio $\left(\mathbb{D},{ }^{1,2}\right.$ Federico Ambrogi $\left(\mathbb{D},{ }^{3}\right.$ Massimo de Cal $\left(\mathbb{D},{ }^{4,5}\right.$ Elena Vianello $\left(\mathbb{D},{ }^{1}\right.$ \\ Claudio Ronco, ${ }^{4,5}$ and Massimiliano M. Corsi Romanelli $\mathbb{I}^{1,6}$ \\ ${ }^{1}$ Department of Biomedical Sciences for Health, Laboratory of Clinical Pathology, Università degli Studi di Milano, Milan, Italy \\ ${ }^{2}$ I.R.C.C.S. Policlinico San Donato, San Donato Milanese, Milan, Italy \\ ${ }^{3}$ Department of Clinical Sciences and Community Health, Laboratory of Medical Statistics, Biometry and Epidemiology \\ "G.A. Maccaro", Università degli Studi di Milano, Milan, Italy \\ ${ }^{4}$ Department of Nephrology, Dialysis \& Transplantation, San Bortolo Hospital, Vicenza, Italy \\ ${ }^{5}$ International Renal Research Institute Vicenza (IRRIV), San Bortolo Hospital, Vicenza, Italy \\ ${ }^{6}$ Service of Laboratory Medicine 1-Clinical Pathology, I.R.C.C.S. Policlinico San Donato, San Donato Milanese, Milan, Italy \\ Correspondence should be addressed to Elena Dozio; elena.dozio@unimi.it
}

Received 25 July 2018; Accepted 20 September 2018; Published 15 October 2018

Academic Editor: Jacek Cezary Szepietowski

Copyright (C) 2018 Elena Dozio et al. This is an open access article distributed under the Creative Commons Attribution License, which permits unrestricted use, distribution, and reproduction in any medium, provided the original work is properly cited.

\begin{abstract}
End-stage renal disease patients on dialysis (CKD-G5D) have a high mortality rate due to cardiovascular diseases (CVD). In these patients, inflammation, oxidative stress, and uremia increase the production of glycation products (AGEs) which in turn accelerate CVD onset and progression. Recently, attention has been given to the soluble receptor for AGEs (sRAGE) as a marker of inflammation, oxidative stress, atherosclerosis, and heart failure in CKD-G5D. However, its association with patient outcomes is still under debate. Our aim is to explore whether sRAGE may be a predictor of mortality in CKD-G5D. We studied 123 CKD-G5D for 24 months. Of these patients, 56 were on hemodialysis (HD) and 67 on peritoneal dialysis (PD). Demographic, anthropometric, biochemical, and clinical data were recorded. sRAGE was quantified by enzyme-linked immunosorbent assay. sRAGE was a predictor of mortality at 2 -year follow-up. Each increase of $100 \mathrm{pg} / \mathrm{mL}$ in sRAGE levels was associated with an approximately $7 \%$ increased risk of mortality. Furthermore, in the entire study group, as well as in PD and HD patient subgroups, sRAGE was positively correlated with brain natriuretic peptide (BNP) levels. Mortality rates as well as sRAGE levels in patients who died did not differ between PD and HD patients. In conclusion, the positive association observed with BNP levels suggests a role for sRAGE as a prognostic factor for mortality in CKD-G5D patients displaying an active process of cardiac remodeling.
\end{abstract}

\section{Introduction}

End-stage renal disease (ESRD) patients on dialysis (CKD-G5D) have a high mortality rate due to cardiovascular diseases (CVD), infections, and malnutrition. In addition to traditional CVD risk factors, such as hypertension, diabetes mellitus (DM), and dyslipidemia, which are common in these patients, excessive oxidative stress and chronic inflammation may further increase cardiovascular morbidity and mortality $[1,2]$.
Products of glycation (AGEs) derive from glycation and oxidation of proteins, lipids, and nucleic acids. AGEs increase in conditions of hyperglycemia, oxidative stress, and uremia and in turn promote inflammation, reactive oxygen/nitrogen species production, apoptosis, and metabolic dysfunctions. In this way, AGEs may accelerate CVD onset and progression [3-5].

In the field of CVD, recent attention has been given to the receptor for AGEs (RAGE), a membrane receptor which mediates the damaging effects of these compounds. Besides 
the cell membrane form, RAGE also exists as a soluble molecule, sRAGE. This soluble receptor, by binding AGEs and other RAGE ligands in the circulation, prevents intracellular RAGE signaling and related proinflammatory effects [6]. In cardiometabolic diseases, low rather than high sRAGE levels can be observed, thus reinforcing the idea of a protective role for this molecule $[7,8]$. However, DM and/or ESRD patients, groups with a high risk of CVD, show increased sRAGE levels [9-11]. This phenomenon may be strongly related to inflammation and oxidative stress which promote the expression of RAGE on the cell membrane and its cleavage by metalloproteinases (MMPs) [12-14]. Moreover, in ESRD, the accumulation of sRAGE is also contributed by reduced renal function [15].

Despite a link between levels of sRAGE with parameters of inflammation, oxidative stress, atherosclerosis, and heart failure (HF) in CKD-G5D [16-20], prior studies failed to observe a strong association with patient outcomes [21-24]. However, there are a limited number of studies in this field, and they are difficult to compare due to differences in study design and patient features. In particular, most studies have been focused on accompanying atherosclerosis and vascular calcification as a part of ESRD [21-24].

Since we have recently described a role for sRAGE as a marker of cardiac remodeling in a group of CKD-G5D patients [8], we evaluated the prognostic role of sRAGE in this well-characterized cohort which include both hemodialysis (HD) and peritoneal dialysis (PD) patients. We hypothesized that in those patients with active cardiac remodeling, elevations in sRAGE may have a prognostic significance, and moreover, sRAGE may be a future target for beneficial therapies.

\section{Materials and Methods}

2.1. Source Population. In this prospective, observational cohort study, we analyzed 123 patients on various dialysis modalities. Of these, 56 were on HD and 67 on PD. To be enrolled in the study, patients had to (1) be on HD or PD treatment for at least 3 months, (2) agree to participate in the study with written inform consent, and (3) be $\geq 18$ years. Patients with missing or incomplete clinical history, inability to provide consent, and hepatic encephalopathy were excluded. The observation period was 24 months. During the follow-up period, 23 patients died. This study was performed in accordance with the Declaration of Helsinki, as revised in 2013. The protocol was approved by the Ethics Committee of San Bortolo Hospital (N.41/14).

2.2. Clinical and Laboratory Parameters. Demographic, anthropometric, biochemical, and clinical data (i.e., age, gender, smoking status, alcohol consumption, hypertension, DM, and CVD) were collected. Blood samples were collected during outpatient visits for PD patients or prior to hemodialysis treatment after the long interdialytic interval. Samples for nonroutine assays were collected in EDTA. Plasma was separated by centrifugation, immediately frozen at $-80^{\circ} \mathrm{C}$, and stored until measurements. Routine biochemical parameters were assessed as previously described [20]. sRAGE was measured by a commercial human ELISA kit (R\&D Systems, Minneapolis, MN, USA) according to the manufacturer's instructions. The minimum detectable concentrations ranged from 1.23 to $16.14 \mathrm{pg} / \mathrm{mL}$. The maximum intra- and interassay coefficients of variations were, respectively, $4.8 \%$ and $8.3 \%$. Samples have been assayed in duplicate. The GloMax $^{\circledR}$-Multi Microplate Multimode Reader was used for photometric measurements (Promega, Milan, Italy).

2.3. Statistical Analysis. Quantitative variables are expressed as mean with standard deviation (SD) and median with interquartile range. Qualitative variables are summarized as numbers and percentages. The normality of data distribution was assessed by the Kolmogorov-Smirnoff test. Comparison between two groups was performed by MannWhitney test for continuous variables and $\chi^{2}$ test for nominal variables. The potential univariate association between sRAGE and the selected variables was performed with Pearson's (for normal-distributed data) or Spearman's (for non-normal-distributed data) correlation tests.

The association of sRAGE with mortality in HD and PD patients was evaluated with logistic regression considering possible nonlinear effects using restricted cubic splines with 3 knots. The association was then adjusted considering other possible confounding factors taking into account the number of events. To select possible confounding variables among those available (age, gender, body mass index (BMI), smoking, alcohol, DM, hypertension, CVD, total protein, urea, triglycerides, LDL-cholesterol, HDL-cholesterol, brain natriuretic peptide (BNP), and glucose), a LASSO penalized logistic regression was used. Regression analyses are represented as OD and 95\% CI. Differences were considered significant at $p<0.05$ (95\% CI).

\section{Results}

3.1. Baseline Characteristics of Patients. The baseline characteristics of the patients included in the study are presented in Table 1. There was no evidence of difference in the mean age of HD and PD groups as well in the existing comorbid conditions. Concerning anthropometric and biochemical parameters, it is noteworthy that PD patients had higher body BMI and increased levels of alanine aminotransferase (ALT), aspartate aminotransferase (AST), LDL-cholesterol, total cholesterol, and urea levels in comparison with HD patients. Moreover, PD patients had lower uric acid, BNP, and albumin levels. Also, sRAGE concentration was lower in PD patients. No significant difference was observed in total protein and C-reactive protein (CRP) levels. The number of patients who died during the follow-up period was 23. Of these, 10 were $\mathrm{HD}$ and 13 were PD patients with similar percentage of death between the two groups. There was no significant difference in the levels of sRAGE between HD and PD patients who died $(4597.38 \pm 930.18 \mathrm{pg} / \mathrm{mL}$ vs. $3480.86 \pm 1538.62 \mathrm{pg} / \mathrm{mL} ; p=0.07)$.

3.2. Univariate Association of sRAGE with Clinical Parameters. The results of the correlation analyses are shown in Table 2. In the whole group of patients, sRAGE was 


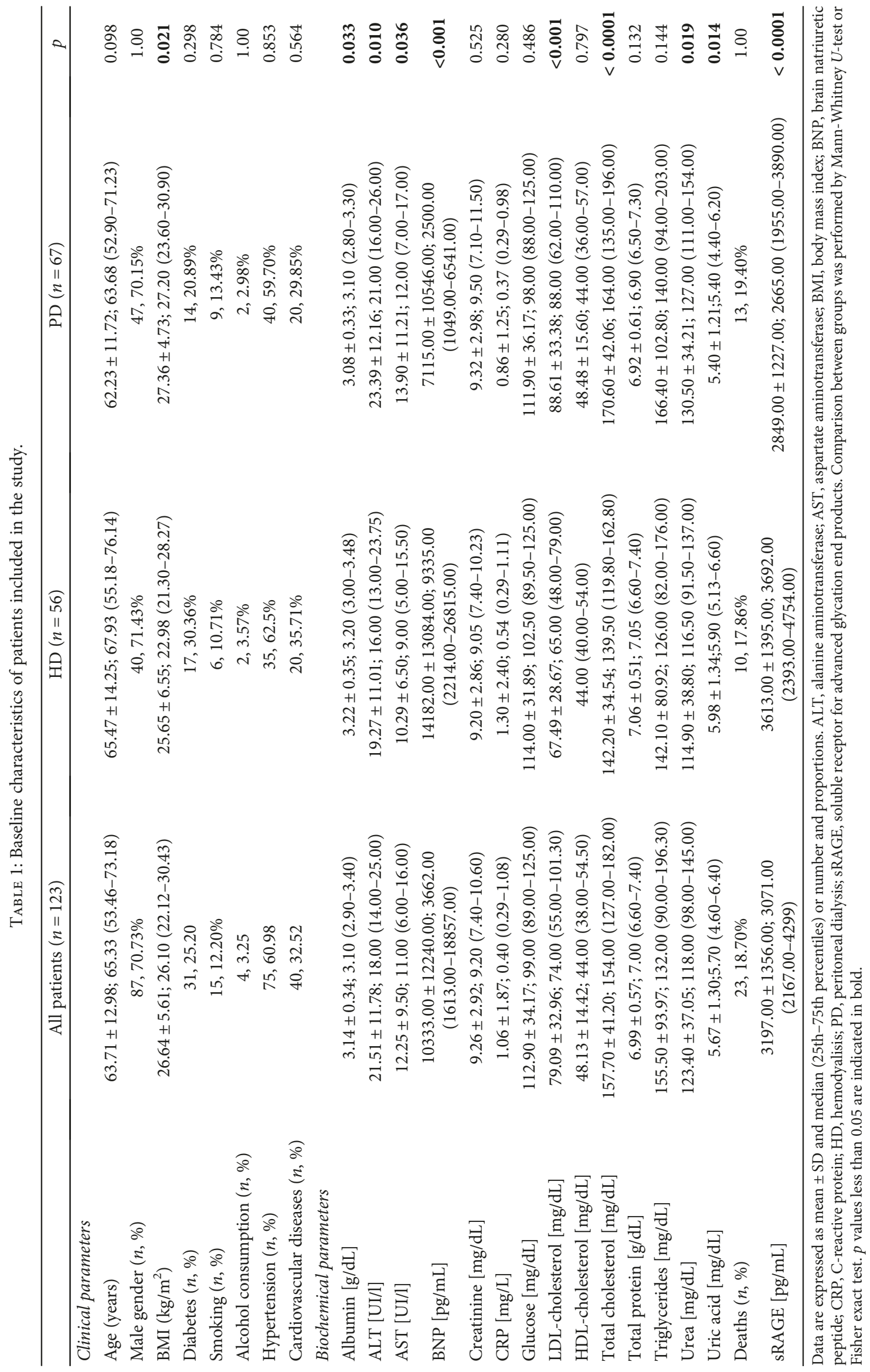


TABLE 2: Correlations of sRAGE with baseline characteristics of patients included in the study analyzed as a whole group and after classification according to the dialytic treatment.

\begin{tabular}{|c|c|c|c|}
\hline sRAGE & $\begin{array}{c}\text { All patients } \\
r\end{array}$ & $\begin{array}{c}\mathrm{HD} \\
r\end{array}$ & $\begin{array}{c}\mathrm{PD} \\
r\end{array}$ \\
\hline Age (years) & $0.073^{\circ}$ & 0.031 & $0.001^{\circ}$ \\
\hline Albumin $[\mathrm{g} / \mathrm{dL}]$ & $-0.071^{\circ}$ & -0.108 & -0.134 \\
\hline ALT [UI/l] & $-0.029^{\circ}$ & $0.064^{\circ}$ & $0.094^{\circ}$ \\
\hline AST [UI/l] & $-0.077^{\circ}$ & $-0.163^{\circ}$ & $0.135^{\circ}$ \\
\hline BMI $\left(\mathrm{kg} / \mathrm{m}^{2}\right)$ & $-0.010^{\circ}$ & $0.146^{\circ}$ & -0.037 \\
\hline $\mathrm{BNP}[\mathrm{pg} / \mathrm{mL}]$ & $0.353^{* * * \circ}$ & $0.281{ }^{* \circ}$ & $0.334^{* * \circ}$ \\
\hline Creatinine $[\mathrm{mg} / \mathrm{dL}]$ & 0.077 & $0.060^{\circ}$ & 0.067 \\
\hline $\mathrm{CRP}[\mathrm{mg} / \mathrm{L}]$ & $0.006^{\circ}$ & $0.156^{\circ}$ & $-0.241 *$ \\
\hline Glucose $[\mathrm{mg} / \mathrm{dL}]$ & $-0.049^{\circ}$ & $-0.081^{\circ}$ & $-0.075^{\circ}$ \\
\hline HDL-cholesterol [mg/dL] & $-0.019^{\circ}$ & $-0.200^{\circ}$ & $0.113^{\circ}$ \\
\hline LDL-cholesterol [mg/dL] & $-0.365^{* * *}$ & $-0.433^{* * *}$ & -0.210 \\
\hline Total cholesterol [mg/dL] & $-0.339^{* * *}$ & $-0.456^{* * *}$ & -0.130 \\
\hline Total protein $[\mathrm{g} / \mathrm{dL}]$ & -0.056 & $-0.133^{\circ}$ & -0.070 \\
\hline Triglycerides [mg/dL] & $-0.127^{\circ}$ & $-0.111^{\circ}$ & $-0.069^{\circ}$ \\
\hline Uric acid [mg/dL] & 0.087 & 0.115 & -0.067 \\
\hline Urea $[\mathrm{mg} / \mathrm{dL}]$ & -0.115 & 0.067 & -0.196 \\
\hline
\end{tabular}

ALT, alanine aminotransferase; AST, aspartate aminotransferase; BMI, body mass index; BNP, brain natriuretic peptide; CRP, C-reactive protein; HD, hemodyalisis; PD, peritoneal dialysis; sRAGE, soluble receptor for advanced glycation end products. Correlations were evaluated with Spearman's $\left({ }^{\circ}\right)$ or Pearson's correlation coefficient, as appropriate. ${ }^{*} p<0.05 ;{ }^{* *} p<0.01$; ${ }^{* * *} p<0.0001$

positively correlated with $\mathrm{BNP}(r=0.353, p<0.001)$ and negatively with LDL-cholesterol $(r=-0.365, p<0.001)$ and total cholesterol $(r=-0.339, p<0.001)$ levels. Similarly, in the HD group, sRAGE was positively correlated with BNP $(r=0.281, p<0.05)$ and negatively with LDL-cholesterol $(r=-0.433, p<0.001)$ and total cholesterol $(r=-0.456$, $p<0.001)$. In the PD group, we observed a positive correlation of sRAGE with BNP $(r=0.334, p<0.01)$ and a negative correlation with CRP $(r=-0.241, p<0.05)$ levels.

3.3. Association of sRAGE Levels with Mortality. There was no evidence of nonlinear effect for the association of sRAGE with mortality ( $p=0.33$ ); therefore, only the linear effect was retained. According to the LASSO penalized logistic regression, the following variables were selected and then utilized for the multivariable regression model: age, BNP, glucose, HDL-cholesterol, smoking, and sRAGE. In particular, three regression models are reported (Table 3 ): model 1 with only sRAGE (univariate); model 2 adjusted for age, gender, glucose, and HDL-cholesterol; and model 3 adjusted also for BNP. sRAGE was associated with mortality in models 1 and $2(p=0.016$ and $p=0.006$, respectively). Considering model 2 , a difference of $100 \mathrm{pg} / \mathrm{mL}$ in the value of sRAGE concentrations corresponds to a difference of about $7 \%$ in the probability of death. In model 3, which further includes BNP as the adjustment factor, the statistical significance was lost $(p=0.096)$ (Table 3$)$. We also observed an association between BNP and mortality in these patients with
TABLE 3: Adjusted odds ratio for overall mortality according to sRAGE levels.

\begin{tabular}{lcc}
\hline Model & OR (95\% CI) & $p$ value \\
\hline $\begin{array}{l}\text { (1) Univariate } \\
\begin{array}{l}\text { (2) Adjusted for age, gender, } \\
\text { glucose, and HDL-cholesterol }\end{array}\end{array}$ & $1.044(1.009-1.083)$ & $\mathbf{0 . 0 1 6}$ \\
$\begin{array}{l}\text { (3) Adjusted for age, gender, } \\
\text { glucose, HDL-cholesterol, and BNP }\end{array}$ & $1.042(0.994-1.122)$ & $\mathbf{0 . 0 0 6}$ \\
\hline
\end{tabular}

OR, odds ratio; CI, confidence interval; sRAGE, soluble receptor for advanced glycation end products. Odds ratios are for increment of 100 in the value of sRAGE. $p$ values less than 0.05 are indicated in bold.

an odds ratio (OR) of 1.004 (95\% confidence interval (CI): 1.001-1.008, $p=0.011$ ). The association lost significance after adjusting for age, gender, glucose, HDL-cholesterol, and sRAGE (OR: 1.004; 95\% CI: 0.999-1.009; $p=0.080$ ).

\section{Discussion}

Our findings suggest for the first time a potential role for sRAGE as a prognostic factor for mortality at 2-year followup in a group of CKD-G5D including both $\mathrm{HD}$ and $\mathrm{PD}$ patients. In detail, an increase of $100 \mathrm{pg} / \mathrm{mL}$ in sRAGE levels was associated with a significant increase in the risk of mortality of about $7 \%$.

Moreover, although the concentration of sRAGE was higher in the $\mathrm{HD}$ than in the PD group, we observed that the rate of mortality as well as sRAGE levels in patients who died did not differ between the two types of dialysis modalities.

The role of sRAGE as a predictor of mortality in patients on dialysis is still under debate. The few studies that have been performed in this field up until now failed to observe any association [22, 24-26]. Only a single study observed a trend, although not statistically significant, towards increased mortality with higher rather than lower sRAGE levels [25]. While not completely clear, the reasons for such differences between our and other studies may relate to differences in the clinical characteristics of the patients enrolled.

In our patients, we observed an increase in BNP concentrations and a strong positive correlation with sRAGE levels. This result may relate to the finding that both $\mathrm{BNP}$ and NT-pro-BNP have been previously described to be elevated in CKD-G5D patients, and it is well known that they are strongly associated with left ventricular hypertrophy and systolic dysfunction and have a predictive potential for HF and mortality [27-30]. Most of the previous studies on sRAGE and mortality in CKD-G5D have been performed focusing on atherosclerosis and vascular calcification as the main CVD outcomes, while they did not explore specific parameters of cardiac remodeling and $\mathrm{HF}$ as well as their association with sRAGE $[22,24,26]$. Our study is the first one which highlights the existence of an increased mortality rate with higher sRAGE levels in patients with a greater increase in BNP, thus suggesting a potential role for sRAGE as a predictor of mortality most specifically in patients with a significant deterioration of heart function. To be noted, according to the LASSO penalized logistic regression, sRAGE and BNP were 
just two of the markers that were selected as potential variables related to mortality in our patients. Moreover, when $\mathrm{BNP}$ was included as the adjustment factor in the regression model, sRAGE lost the statistical significance as a predictor of mortality. These data seem to suggest that sRAGE and BNP could be related and could reflect the same outcome, but we cannot exclude that the lack of significance may be also due to a limited number of events observed in our population. Our hypothesis seems to be strongly supported by results obtained in other types of patients in which increasing sRAGE levels emerged as a marker of worsening heart function and mortality [31-35]. These studies, in fact, indicated the existence of an association between sRAGE and the severity of HF, whereby sRAGE levels are increased in patients with higher BNP levels. Additional studies in larger cohorts of patients and the inclusion of echocardiographic parameters could help to confirm these results.

A novel finding of our study is also the observation that sRAGE levels are higher in HD than in PD patients. Such a comparison has been previously performed only in the study by Isoyama et al. [25]. These authors observed no difference between the two groups not only in sRAGE levels but also in the other clinical parameters evaluated. The reasons why the levels of sRAGE are different in HD and PD patients could be related to the way the two treatments retain or remove the solutes and the presence of a residual renal function that probably confers some advantages to PD patients. However, it is also possible that specific clinical factors, including but not limited to body composition, nutritional status, inflammatory, and prooxidant factors, may affect the circulating molecules [36-38]. More studies are needed in this regard.

Besides being a biomarker, sRAGE is a bioactive molecule with important physiological and pathological functions. The putative role of membrane RAGE activation and sRAGE circulating levels in cardiovascular complications of $\mathrm{CKD}$ patients are still very controversial. Previous studies indicated that AGEs levels are higher in CKD patients and, by interacting with membrane RAGE, they may activate intracellular signaling pathways that lead to oxidative stress, inflammation, vascular stiffness, atherosclerosis, tissue remodeling, and fibrosis [39-41]. Blockage of RAGE has been suggested as one potential protective mechanism against AGE-induced cardiovascular complications and sRAGE may just work in this way by linking to AGEs and preventing their interaction with RAGE [6, 40, 42]. These observations seem to be in contradiction with our findings that showed an increased mortality associated with the upregulation of sRAGE levels. In this regard, it is important to recall that sRAGE is a circulating pool composed of two different forms, namely esRAGE, the endogenous secretory form, and cRAGE that derives from proteolytic cleavage of the membrane-bound molecule, and that they can have different roles. Among these forms, esRAGE seems to be the real decoy receptor with important protective functions, whereas cRAGE appears more as a surrogate marker of inflammation. Previous observations indicated that inflammation- and oxidative stress-related conditions are associated with a decrease in esRAGE levels promoted by RAGE activation and an increased cleavage of the surface RAGE induced by MMPs and other enzymes $[12-14,43-45]$. The quantification of the two forms that form the circulating pool could probably help to shed more light on the real role of sRAGE in different pathological conditions. Unfortunately, we have not determined which type of sRAGE is increased in our patients. However, based on previous results, we expect to observe a major contribution by cRAGE. Accordingly, it is possible that the increased levels of sRAGE observed in HD patients compared to PD may be due partly to the presence of residual renal function in PD and partly to the increased inflammation and oxidative stress described in HD patients, two conditions that may increase cRAGE production $[2,46]$. Noteworthy also is our observation of an inverse correlation between sRAGE and CRP levels in PD but not in HD patients. This data could thus be interpreted as a failed protective response of sRAGE in HD probably due to the increased production of cRAGE and the activation of a loop which further promotes inflammation, RAGE cleavage, and esRAGE downregulation. However, the real biological role of the different sRAGE forms in these patients remains to be elucidated.

Several limitations of this study should be acknowledged, starting with the number of patients included and the followup limited to 2 years that did not allow us to observe more events. Moreover, the availability of some additional clinical parameters, such as echocardiography data on heart function, inflammatory biomarkers, and sRAGE isoforms, would allow us to obtain a more unbiased estimation of the relations observed and also to confirm the inflammation milieu previously described in HD [2, 46].

\section{Conclusions}

In summary, our results revealed for the first time that in patients with active cardiac remodeling, elevations in sRAGE may have a prognostic significance. Although we cannot conclude whether sRAGE is a predictor of HF, its quantifications seem to be as a useful supportive tool that could help to identify high-risk patients. Whether sRAGE might also be a future target for beneficial therapies in these patients needs to be explored in the future.

\section{Data Availability}

The data used to support the findings of this study are available from the corresponding author upon request.

\section{Conflicts of Interest}

The authors declare that there is no conflict of interest regarding the publication of this article.

\section{Authors' Contributions}

Research idea and study design were developed by ED and FA; data acquisition was handled by $\mathrm{MdC}$ and $\mathrm{EV}$; data analysis/interpretation was handled by ED and FA; statistical analysis was conducted by ED and FA; the study was 
supervised by CR; mentorship was handled by MMCR. All authors revised and approved the final version of the article. Elena Dozio and Federico Ambrogi equally contributed to the manuscript.

\section{Acknowledgments}

The authors wish to thank Ms. Judith D. Baggott for language editing.

\section{References}

[1] C. Wanner, K. Amann, and T. Shoji, "The heart and vascular system in dialysis," Lancet, vol. 388, no. 10041, pp. 276-284, 2016.

[2] V. Liakopoulos, S. Roumeliotis, X. Gorny, E. Dounousi, and P. R. Mertens, "Oxidative stress in hemodialysis patients: a review of the literature," Oxidative Medicine and Cellular Longevity, vol. 2017, Article ID 3081856, 22 pages, 2017.

[3] T. Miyata, Y. Wada, Z. Cai et al., "Implication of an increased oxidative stress in the formation of advanced glycation end products in patients with end-stage renal failure," Kidney International, vol. 51, no. 4, pp. 1170-1181, 1997.

[4] P. Stenvinkel, J. J. Carrero, J. Axelsson, B. Lindholm, O. Heimburger, and Z. Massy, "Emerging biomarkers for evaluating cardiovascular risk in the chronic kidney disease patient: how do new pieces fit into the uremic puzzle?," Clinical Journal of the American Society of Nephrology, vol. 3, no. 2, pp. 505-521, 2008.

[5] D. Deluyker, L. Evens, and V. Bito, "Advanced glycation end products (AGEs) and cardiovascular dysfunction: focus on high molecular weight AGEs," Amino Acids, vol. 49, no. 9, pp. 1535-1541, 2017.

[6] N. Mahajan and V. Dhawan, "Receptor for advanced glycation end products (RAGE) in vascular and inflammatory diseases," International Journal of Cardiology, vol. 168, no. 3, pp. 17881794, 2013.

[7] E. Selvin, M. K. Halushka, A. M. Rawlings et al., "sRAGE and risk of diabetes, cardiovascular disease, and death," Diabetes, vol. 62, no. 6, pp. 2116-2121, 2013.

[8] E. Dozio, S. Briganti, A. Delnevo et al., "Relationship between soluble receptor for advanced glycation end products (sRAGE), body composition and fat distribution in healthy women," European Journal of Nutrition, vol. 56, no. 8, pp. 2557-2564, 2017.

[9] M. Challier, S. Jacqueminet, O. Benabdesselam, A. Grimaldi, and J. L. Beaudeux, "Increased serum concentrations of soluble receptor for advanced glycation endproducts in patients with type 1 diabetes," Clinical Chemistry, vol. 51, no. 9, pp. 17491750, 2005.

[10] M. Kalousová, M. Hodková, M. Kazderová et al., "Soluble receptor for advanced glycation end products in patients with decreased renal function," American Journal of Kidney Diseases, vol. 47, no. 3, pp. 406-411, 2006.

[11] R. Meerwaldt, C. J. Zeebregts, G. Navis, J. L. Hillebrands, J. D. Lefrandt, and A. J. Smit, "Accumulation of advanced glycation end products and chronic complications in ESRD treated by dialysis," American Journal of Kidney Diseases, vol. 53, no. 1, pp. 138-150, 2009.

[12] K. Nakamura, S. Yamagishi, H. Adachi et al., "Serum levels of sRAGE, the soluble form of receptor for advanced glycation end products, are associated with inflammatory markers in patients with type 2 diabetes," Molecular Medicine, vol. 13, no. 3-4, pp. 185-189, 2007.

[13] N. Yamakawa, T. Uchida, M. A. Matthay, and K. Makita, "Proteolytic release of the receptor for advanced glycation end products from in vitro and in situ alveolar epithelial cells," American Journal of Physiology. Lung Cellular and Molecular Physiology, vol. 300, no. 4, pp. L516-L525, 2011.

[14] Y. Izushi, K. Teshigawara, K. Liu et al., "Soluble form of the receptor for advanced glycation end-products attenuates inflammatory pathogenesis in a rat model of lipopolysaccharide-induced lung injury," Journal of Pharmacological Sciences, vol. 130, no. 4, pp. 226-234, 2016.

[15] K. Prasad, I. Dhar, Q. Zhou, H. Elmoselhi, M. Shoker, and A. Shoker, "AGEs/sRAGE, a novel risk factor in the pathogenesis of end-stage renal disease," Molecular and Cellular Biochemistry, vol. 423, no. 1-2, pp. 105-114, 2016.

[16] J. K. Kim, S. Park, M. J. Lee et al., "Plasma levels of soluble receptor for advanced glycation end products (sRAGE) and proinflammatory ligand for RAGE (EN-RAGE) are associated with carotid atherosclerosis in patients with peritoneal dialysis," Atherosclerosis, vol. 220, no. 1, pp. 208-214, 2012.

[17] H. S. Kim, W. Chung, A. J. Kim et al., "Circulating levels of soluble receptor for advanced glycation end product are inversely associated with vascular calcification in patients on haemodialysis independent of S100A12 (EN-RAGE) levels," Nephrology (Carlton), vol. 18, no. 12, pp. 777-782, 2013.

[18] G. S. M. El-Saeed, F. Fadel, M. F. Elshamaa et al., “Advanced glycation end products and soluble receptor as markers of oxidative stress in children on hemodialysis," Renal Failure, vol. 37, no. 9, pp. 1452-1456, 2015.

[19] M. Lazo, M. K. Halushka, L. Shen et al., "Soluble receptor for advanced glycation end products and the risk for incident heart failure: the Atherosclerosis Risk in Communities Study," American Heart Journal, vol. 170, no. 5, pp. 961-967, 2015.

[20] E. Dozio, V. Corradi, E. Vianello et al., "Increased levels of sRAGE in diabetic CKD-G5D patients: a potential protective mechanism against AGE-related upregulation of fibroblast growth factor 23 and inflammation," Mediators of Inflammation, vol. 2017, Article ID 9845175, 9 pages, 2017.

[21] M. Kalousova, M. Jachymova, O. Mestek et al., "Receptor for advanced glycation end products-soluble form and gene polymorphisms in chronic haemodialysis patients," Nephrology, Dialysis, Transplantation, vol. 22, no. 7, pp. 2020-2026, 2007.

[22] A. Nakashima, J. J. Carrero, A. R. Qureshi et al., "Effect of circulating soluble receptor for advanced glycation end products (sRAGE) and the proinflammatory RAGE ligand (EN-RAGE, S100A12) on mortality in hemodialysis patients," Clinical Journal of the American Society of Nephrology, vol. 5, no. 12, pp. 2213-2219, 2010.

[23] N. Isoyama, A. Machowska, A. R. Qureshi et al., "Elevated circulating S100A12 associates with vascular disease and worse clinical outcome in peritoneal dialysis patients," Peritoneal Dialysis International, vol. 36, no. 3, pp. 269-276, 2016.

[24] E. S. Jung, W. Chung, A. J. Kim et al., "Associations between soluble receptor for advanced glycation end products (sRAGE) and S100A12 (EN-RAGE) with mortality in long-term hemodialysis patients," Journal of Korean Medical Science, vol. 32, no. 1, pp. 54-59, 2017.

[25] N. Isoyama, P. Leurs, A. R. Qureshi et al., "Plasma S100A12 and soluble receptor of advanced glycation end product levels and mortality in chronic kidney disease stage 5 patients," 
Nephrology, Dialysis, Transplantation, vol. 30, no. 1, pp. 84-91, 2015.

[26] B. H. Choi, H. Ro, E. S. Jung et al., "Circulating S100A12 levels are associated with progression of abdominal aortic calcification in hemodialysis patients," PLoS One, vol. 11, no. 2, article e0150145, 2016.

[27] F. Mallamaci, C. Zoccali, G. Tripepi et al., "Diagnostic potential of cardiac natriuretic peptides in dialysis patients," Kidney International, vol. 59, no. 4, pp. 1559-1566, 2001.

[28] L. H. Madsen, S. Ladefoged, P. Corell, M. Schou, P. R. Hildebrandt, and D. Atar, "N-terminal pro brain natriuretic peptide predicts mortality in patients with end-stage renal disease in hemodialysis," Kidney International, vol. 71, no. 6, pp. 548-554, 2007.

[29] S. Satyan, R. P. Light, and R. Agarwal, "Relationships of $\mathrm{N}$-terminal pro-B-natriuretic peptide and cardiac troponin $\mathrm{T}$ to left ventricular mass and function and mortality in asymptomatic hemodialysis patients," American Journal of Kidney Diseases, vol. 50, no. 6, pp. 1009-1019, 2007.

[30] A. Y.-M. Wang, C. W. K. Lam, C. M. Yu et al., "N-terminal pro-brain natriuretic peptide: an independent risk predictor of cardiovascular congestion, mortality, and adverse cardiovascular outcomes in chronic peritoneal dialysis patients," Journal of the American Society of Nephrology, vol. 18, no. 1, pp. 321-330, 2006.

[31] S. Raposeiras-Roubín, B. K. Rodiño-Janeiro, L. GrigorianShamagian et al., "Relation of soluble receptor for advanced glycation end products to predict mortality in patients with chronic heart failure independently of Seattle Heart Failure Score," The American Journal of Cardiology, vol. 107, no. 6, pp. 938-944, 2011.

[32] P. Boschetto, I. Campo, M. Stendardo et al., "Plasma sRAGE and $\mathrm{N}$-(carboxymethyl) lysine in patients with $\mathrm{CHF}$ and/or COPD," European Journal of Clinical Investigation, vol. 43, no. 6, pp. 562-569, 2013.

[33] H. Brodska, K. Malickova, J. Valenta, A. Fabio, and T. Drabek, "Soluble receptor for advanced glycation end products predicts 28-day mortality in critically ill patients with sepsis," Scandinavian Journal of Clinical and Laboratory Investigation, vol. 73, no. 8, pp. 650-660, 2013.

[34] T. F. Lancefield, S. K. Patel, M. Freeman et al., "The receptor for advanced glycation end products (RAGE) is associated with persistent atrial fibrillation," PLoS One, vol. 11, no. 9, article e0161715, 2016.

[35] S. G. Wannamethee, P. Welsh, O. Papacosta et al., "Circulating soluble receptor for advanced glycation end product: crosssectional associations with cardiac markers and subclinical vascular disease in older men with and without diabetes," Atherosclerosis, vol. 264, pp. 36-43, 2017.

[36] G. Mircescu, "Oxidative stress: an accomplice to uremic toxicity?," Journal of Renal Nutrition, vol. 16, no. 3, pp. 194-198, 2006.

[37] C.-H. Guoa, C. L. Wangb, P. C. Chen, and T. C. Yang, "Linkage of some trace elements, peripheral blood lymphocytes, inflammation, and oxidative stress in patients undergoing either hemodialysis or peritoneal dialysis," Peritoneal Dialysis International, vol. 31, no. 5, pp. 583-591, 2011.

[38] H. Gokbel, H. Atalay, N. Okudan, Y. Solak, M. Belviranli, and S. Turk, "Coenzyme Q10 and its relation with oxidant and antioxidant system markers in patients with end-stage renal disease," Renal Failure, vol. 33, no. 7, pp. 677-681, 2011.
[39] A. N. Reynolds, A. Duncan, D. Kruimer, and B. J. Venn, "Glycated albumin is associated with body mass index in euglycemic adults but is not predictive of postprandial blood glucose response," Journal of Clinical Laboratory Analysis, vol. 31, no. 5, 2016.

[40] S. Bongarzone, V. Savickas, F. Luzi, and A. D. Gee, “Targeting the receptor for advanced glycation endproducts (RAGE): a medicinal chemistry perspective," Journal of Medicinal Chemistry, vol. 60, no. 17, pp. 7213-7232, 2017.

[41] J. Calviño, S. Cigarran, L. Gonzalez-Tabares et al., “Advanced glycation end products (AGEs) estimated by skin autofluorescence are related with cardiovascular risk in renal transplant," PLoS One, vol. 13, no. 8, article e0201118, 2018.

[42] Y. Liu, M. Yu, Z. Zhang et al., "Blockade of receptor for advanced glycation end products protects against systolic overload-induced heart failure after transverse aortic constriction in mice," European Journal of Pharmacology, vol. 791, pp. 535-543, 2016.

[43] H. Koyama, T. Shoji, H. Yokoyama et al., "Plasma level of endogenous secretory RAGE is associated with components of the metabolic syndrome and atherosclerosis," Arteriosclerosis, Thrombosis, and Vascular Biology, vol. 25, no. 12, pp. 2587-2593, 2005.

[44] L. J. Wang, L. Lu, F. R. Zhang, Q. J. Chen, R. de Caterina, and W. F. Shen, "Increased serum high-mobility group box-1 and cleaved receptor for advanced glycation endproducts levels and decreased endogenous secretory receptor for advanced glycation endproducts levels in diabetic and non-diabetic patients with heart failure," European Journal of Heart Failure, vol. 13, no. 4, pp. 440-449, 2011.

[45] F. Piarulli, A. Lapolla, E. Ragazzi et al., "Role of endogenous secretory RAGE (esRAGE) in defending against plaque formation induced by oxidative stress in type 2 diabetic patients," Atherosclerosis, vol. 226, no. 1, pp. 252-257, 2013.

[46] K. Yong, G. Dogra, N. Boudville, and W. Lim, "Increased inflammatory response in association with the initiation of hemodialysis compared with peritoneal dialysis in a prospective study of end-stage kidney disease patients," Peritoneal Dialysis International, vol. 38, no. 1, pp. 18-23, 2018. 


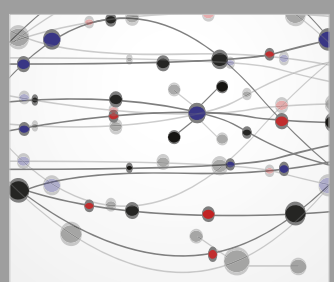

The Scientific World Journal
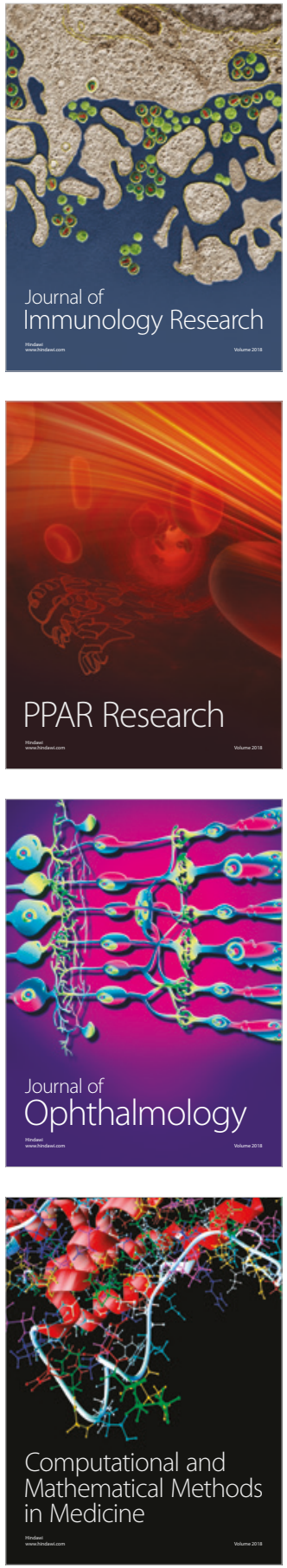

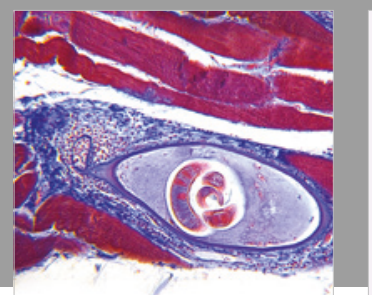

Gastroenterology Research and Practice

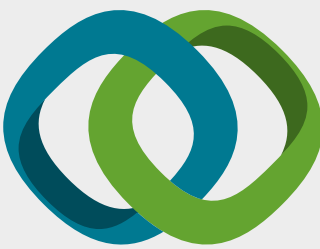

\section{Hindawi}

Submit your manuscripts at

www.hindawi.com
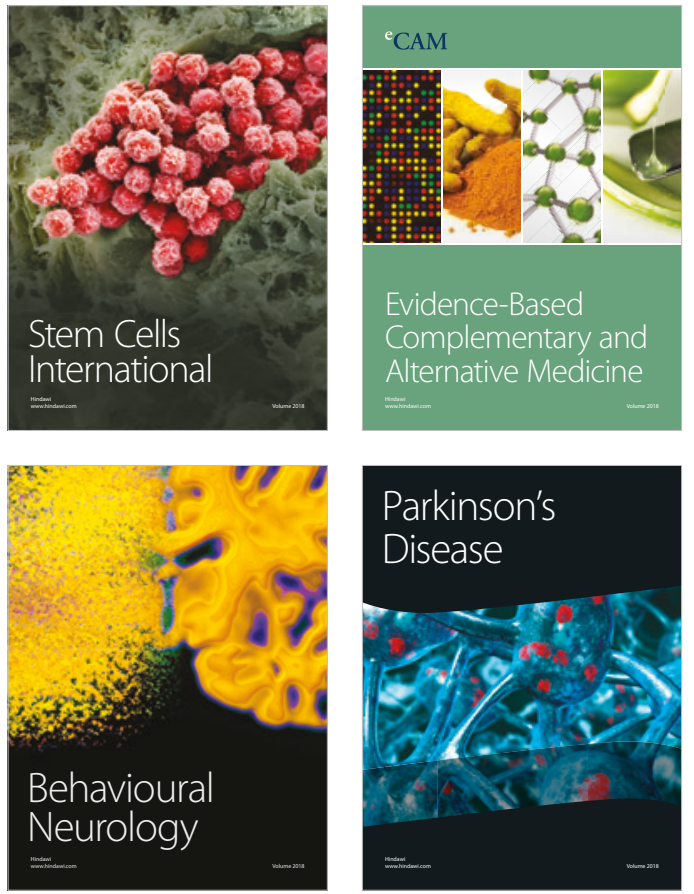

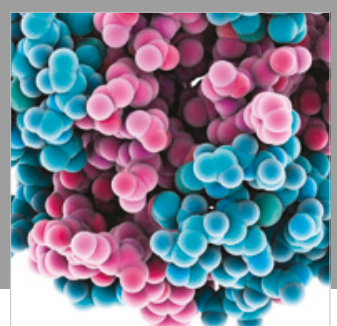

ournal of

Diabetes Research

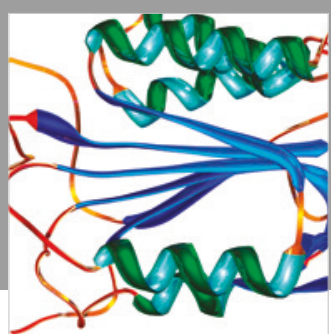

Disease Markers
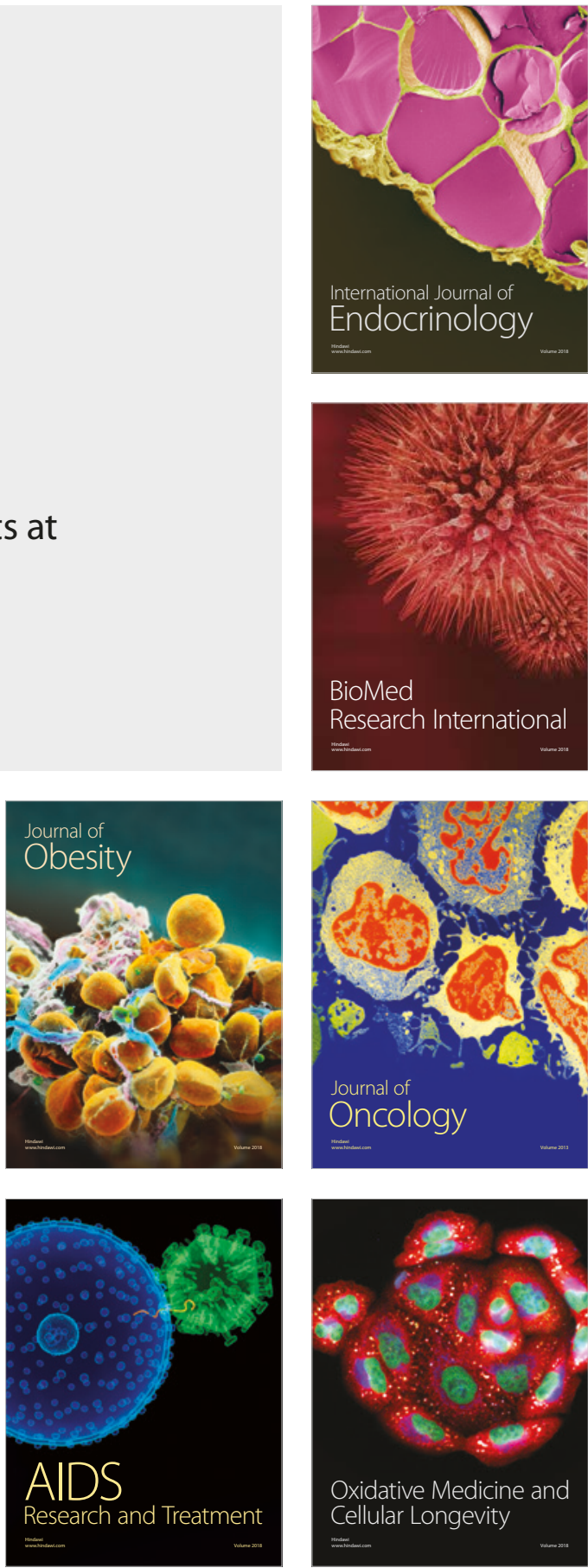